\title{
Simplified derivation of the Hawking-Unruh temperature for an accelerated observer in vacuum
}

\author{
Paul M. Alsing \\ Department of Physics and Astronomy \\ University of New Mexico \\ Albuquerque, New Mexico 87131 \\ alsing@hpc.unm.edu \\ Peter W. Milonni \\ Theoretical Division (T-DOT) \\ Los Alamos National Laboratory \\ Los Alamos, New Mexico 87545 \\ pwm@lanl.gov
}

(Dated: February 1, 2008)

\begin{abstract}
A detector undergoing uniform acceleration $a$ in a vacuum field responds just as though it were immersed in thermal radiation of temperature $T=\hbar a / 2 \pi k c$. A simple, intuitive derivation of this result is given for the case of a scalar field in one spatial dimension. The approach is then extended to treat the case where the field seen by the accelerated observer is a spin-1/2 Dirac field.
\end{abstract}

\section{INTRODUCTION}

Hawking ${ }^{1}$ predicted that a black hole should radiate with a temperature $T=\hbar g / 2 \pi k c$, where $g$ is the gravitational acceleration at the surface of the black hole, $k$ is Boltzmann's constant, and $c$ is the speed of light. This results from the effect of the strong gravitation on the vacuum field. Shortly thereafter it was shown separately by Davies and Unruh that a uniformly accelerated detector in vacuum responds just as though it were in a thermal field 
of temperature $2,3,4,5,6$

$$
T=\frac{\hbar a}{2 \pi k c}
$$

where $a$ is the acceleration in the instantaneous rest frame of the detector. These results suggest profound consequences for the merger of quantum field theory and general relativity and sparked intense debates over unresolved questions that are still actively investigated today: (1) if black holes are not really "black," are naked singularities the ultimate fate of black holes, or will a long-sought fusion of quantum mechanics and general relativity into a coherent theory of quantum gravity prevent such occurrences?; (2) if a quantum mechanical pure state is dropped into a black hole and pure thermal (uncorrelated) radiation results, how does one explain the apparent non-unitary evolution of a pure state to a mixed state?

This intriguing result of quantum field theory has arguably not been derived in any physically intuitive way. Numerous explicit and detailed calculations have appeared in the scientific literature over the last, roughly 30 years for a wide host of spacetimes. However, even for the "simplest" calculation involving a scalar field, the intricacies of field theory techniques, coupled with a forest of special function properties, makes most derivations intractable for the curious, casual scientific non-specialist. An investigation of the Unruh effect for the case of Dirac particles of spin 1/2 brings in a whole host of new machinery, least of which is the formulation of the Dirac equation in curvilinear coordinates (i.e. essentially in curved spacetime). This quickly goes beyond the expertise of most casual scientific readers. However, in both cases, this beautiful and important result can be stated quite simply: for a scalar field (bosons) the accelerated observer sees a Bose-Einstein (BE) distribution at temperature $T$ given by Eq.(1), while for a spin-1/2 field the accelerated observer sees a Fermi-Dirac distribution at the same temperature.

It is the purpose of this paper to present simplified derivations of Eq.(1) in a way that is suitable for advanced undergraduate or beginning graduate students and that elucidates the essential underlying physics of the Unruh effect. Once one accepts the simplest features of a quantized vacuum field, the result Eq.(1) emerges as a consequence of time dependent Doppler shifts in the field seen by the accelerated observer.

In the following section the essential features of uniform acceleration for our purposes 
are reviewed, and in Section 3 we use these results to obtain Eq.(1) in an almost trivial way based on the Doppler effect. In Section 4 this simple approach to the derivation of the temperature Eq.(1) is developed in more detail. In Section 5 we extend the previous calculations for scalar fields to spin-1/2 Dirac fields. We close with a brief summary and discussion.

\section{UNIFORM ACCELERATION}

Uniform acceleration is defined as a constant acceleration $a$ in an instantaneous inertial frame in which the observer is at rest. The acceleration $d v / d t$ in the lab frame is related to $a$ by the Lorentz transformation formula

$$
\frac{d v}{d t}=a\left(1-\frac{v^{2}}{c^{2}}\right)^{3 / 2}
$$

Integrating, and taking $v=0$ at $t=0$, we have $v(t)=a t / \sqrt{1+a^{2} t^{2} / c^{2}}$. The relation $d t=d \tau / \sqrt{1-v^{2} / c^{2}}$ between the lab time $(t)$ and the proper time $(\tau)$ for the accelerated observer gives $t(\tau)=(c / a) \sinh (a \tau / c)$ if we take $t(\tau=0)=0$. The velocity $v$ of the accelerated observer as seen from the lab frame can be expressed in terms of the proper time $\tau$ as

$$
v(\tau)=c \tanh \left(\frac{a \tau}{c}\right) .
$$

A straightforward integration of the above equations yields the well known hyperbolic orbit of the accelerated (Rindler) observer in the $\mathbf{z}$ direction ${ }^{7}$ :

$$
t(\tau)=\frac{c}{a} \sinh \left(\frac{a \tau}{c}\right), \quad z(\tau)=\frac{c^{2}}{a} \cosh \left(\frac{a \tau}{c}\right) .
$$

Throughout this work we consider $a>0$, i.e. the observer accelerates in the $\mathbf{z}$ direction.

\section{INDICATION OF THERMAL EFFECT OF ACCELERATION}

Consider now a plane-wave field of frequency $\omega_{K}$ and wave vector $\mathbf{K}$ parallel or antiparallel to the direction $\mathbf{z}$ along which the observer is accelerated. In the instantaneous rest 
frame of the observer the frequency $\omega_{K}^{\prime}$ of this field is given by the Lorentz transformation formula

$$
\omega_{K}^{\prime}(\tau)=\frac{\omega_{K}-K v(\tau)}{\sqrt{1-v^{2}(\tau) / c^{2}}}=\frac{\omega_{K}\left[1-\tanh \left(\frac{a \tau}{c}\right)\right]}{\sqrt{1-\tanh ^{2}\left(\frac{a \tau}{c}\right)}}=\omega_{K} e^{-a \tau / c} \quad\left(K=+\omega_{K} / c\right)
$$

for $K=+\omega_{K} / c$, i.e., for plane-wave propagation along the direction $\mathbf{z}$ of the observer's acceleration. For propagation in the $-\mathbf{z}$ direction, similarly,

$$
\omega_{K}^{\prime}(\tau)=\omega_{K} e^{a \tau / c} \quad\left(K=-\omega_{K} / c\right)
$$

Note that, for small values of $a \tau, \omega_{K}^{\prime} \cong \omega_{K}(1 \mp a \tau / c)$, the familiar Doppler shift. Equations (5) and (6) involve time-dependent Doppler shifts seen by the accelerated observer.

Because of these Doppler shifts our accelerated observer sees waves with a time-dependent phase $\varphi(\tau) \equiv \int^{\tau} \omega_{K}^{\prime}\left(\tau^{\prime}\right) d \tau^{\prime}=\left(\omega_{K} c / a\right) \exp (a \tau / c)$. We suppose therefore that, for a wave propagating in the $\mathbf{- z}$ direction, for which $\int^{\tau} \omega_{K}^{\prime}\left(\tau^{\prime}\right) d \tau^{\prime}=\left(\omega_{K} c / a\right) \exp (a \tau / c)$, he sees a frequency spectrum $S(\Omega)$ proportional to

$$
\left|\int_{-\infty}^{\infty} d \tau e^{i \Omega \tau} e^{i\left(\omega_{K} c / a\right) e^{a \tau / c}}\right|^{2}
$$

Changing variables to $y=e^{a \tau / c}$, we have

$$
\begin{aligned}
\int_{-\infty}^{\infty} d \tau e^{i \Omega \tau} e^{i\left(\omega_{K} c / a\right) e^{a \tau / c}} & =\frac{c}{a} \int_{0}^{\infty} d y y^{(i \Omega c / a-1)} e^{i\left(\omega_{K} c / a\right) y} \\
& =\frac{c}{a} \Gamma\left(\frac{i \Omega c}{a}\right)\left(\frac{\omega_{K} c}{a}\right)^{-i \Omega c / a} e^{-\pi \Omega c / 2 a}
\end{aligned}
$$

where $\Gamma$ is the gamma function ${ }^{8}$. Then, since $^{9}\left|\Gamma\left(\frac{i \Omega c}{a}\right)\right|^{2}=\pi /[(\Omega c / a) \sinh (\pi \Omega c / a)]$,

$$
\left|\int_{-\infty}^{\infty} d \tau e^{i \Omega \tau} e^{i\left(\omega_{K} c / a\right) e^{a \tau / c}}\right|^{2}=\frac{2 \pi c}{\Omega a} \frac{1}{e^{2 \pi \Omega c / a}-1}
$$

The time-dependent Doppler shift seen by the accelerated observer therefore leads to the Planck factor $\left(e^{\hbar \Omega / k T}-1\right)^{-1}$ with $T=\hbar a / 2 \pi k c$, which is just equation Eq.(1). We obtain the same result in the case of a wave propagating in the $+\mathbf{z}$ direction.

Note that the time-dependent phase can also be obtained directly by considering the standard nonaccelerated Minkowski plane wave $\exp \left[i \varphi_{ \pm}\right] \equiv \exp \left[i\left(K z \pm \omega_{K} t\right)\right]$ and using equations Eq.(4): $\varphi_{ \pm}(\tau)=K z(\tau) \pm \omega_{K} t(\tau)=\left(\omega_{K} c / a\right) \exp ( \pm a \tau / c)$ with $K=\omega_{K} / c^{10,11}$. 


\section{A MORE FORMAL DERIVATION}

The "derivation" of the temperature Eq.(1) just given leaves much to be desired. We have restricted ourselves to a single field frequency $\omega_{K}$, whereas a quantum field in vacuum has components at all frequencies. Moreover we have noted the appearance of the Planck factor but have not actually compared our result to that appropriate to an observer at rest in a thermal field.

To rectify these deficiencies, let us consider a massless scalar field in one spatial dimension $(z)$, quantized in a box of volume $V^{12}$ :

$$
\hat{\phi}=\sum_{K}\left(\frac{2 \pi \hbar c^{2}}{\omega_{K} V}\right)^{1 / 2}\left[\hat{a}_{K} e^{-i \omega_{K} t}+\hat{a}_{K}^{\dagger} e^{i \omega_{K} t}\right] .
$$

Here $K= \pm \omega_{K} / c$, and $\hat{a}_{K}$ and $\hat{a}_{K}^{\dagger}$ are respectively the annihilation and creation operators for mode $K\left(\left[\hat{a}_{K}, a_{K^{\prime}}^{\dagger}\right]=\delta_{K K^{\prime}},\left[\hat{a}_{K}, a_{K^{\prime}}\right]=0\right)$. We use a caret $\left({ }^{\wedge}\right)$ to denote quantummechanical operators. The expectation value $\left\langle(d \hat{\phi} / d t)^{2}\right\rangle / 4 \pi c^{2}$ of the energy density of this field is $V^{-1} \sum_{K} \hbar \omega_{K}\left[\left\langle\hat{a}_{K}^{\dagger} \hat{a}_{K}\right\rangle+1 / 2\right]$. For simplicity we consider the field at a particular point in space (say, $z=0$ ), since spatial variations of the field will be of no consequence for our purposes.

For a thermal state the number operator $\hat{a}_{K}^{\dagger} \hat{a}_{K}$ has the expectation value $\left(e^{\hbar \omega_{K} / k T}-1\right)^{-1}$. Consider the Fourier transform operator

$$
\hat{g}(\Omega)=\frac{1}{2 \pi} \int_{-\infty}^{\infty} d t \hat{\phi} e^{i \Omega t}=\sum_{K}\left(\frac{2 \pi \hbar c^{2}}{\omega_{K} V}\right)^{1 / 2} \hat{a}_{K} \delta\left(\omega_{K}-\Omega\right), \quad \Omega>0 .
$$

The expectation value $\left\langle\hat{g}^{\dagger}(\Omega) \hat{g}\left(\Omega^{\prime}\right)\right\rangle$ in thermal equilibrium is therefore

$$
\begin{aligned}
\left\langle\hat{g}^{\dagger}(\Omega) \hat{g}\left(\Omega^{\prime}\right)\right\rangle & =\sum_{K}\left(\frac{2 \pi \hbar c^{2}}{\omega_{K} V}\right)\left\langle\hat{a}_{K}^{\dagger} \hat{a}_{K}\right\rangle \delta\left(\Omega-\Omega^{\prime}\right) \delta\left(\omega_{K}-\Omega\right) \\
& =\sum_{K}\left(\frac{2 \pi \hbar c^{2}}{\omega_{K} V}\right) \frac{1}{e^{\hbar \omega_{K} / k T}-1} \delta\left(\Omega-\Omega^{\prime}\right) \delta\left(\omega_{K}-\Omega\right) .
\end{aligned}
$$

We go to the limit where the volume of our quantization box becomes very large, $V \rightarrow \infty$, so that we can replace in the usual fashion the sum over $K$ by an integral: $\sum_{K} \rightarrow(V / 2 \pi) \int d K^{13}$. Thus

$$
\left\langle\hat{g}^{\dagger}(\Omega) \hat{g}\left(\Omega^{\prime}\right)\right\rangle=\hbar c^{2} \int_{-\infty}^{\infty} d K \frac{1}{\omega_{K}} \frac{1}{e^{\hbar \Omega / k T}-1} \delta(|K| c-\Omega) \delta\left(\Omega-\Omega^{\prime}\right)
$$




$$
=\frac{2 \hbar c / \Omega}{e^{\hbar \Omega / k T}-1} \delta\left(\Omega-\Omega^{\prime}\right)
$$

Now let us consider an observer in uniform acceleration in the quantized vacuum field. This observer sees each field frequency Doppler-shifted according to Eq.(5) and Eq.(6), and so for him the operator $\hat{g}(\Omega)$ has the form

$$
\begin{aligned}
\hat{g}(\Omega)= & \frac{1}{2 \pi} \int_{-\infty}^{\infty} d \tau e^{i \Omega \tau} \sum_{K}\left(\frac{2 \pi \hbar c^{2}}{\omega_{K} V}\right)^{1 / 2}\left[\hat{a}_{K} e^{-i \int^{\tau} d \tau^{\prime} \omega_{K}^{\prime}\left(\tau^{\prime}\right)}+\hat{a}_{K}^{\dagger} e^{i \int^{\tau} d \tau^{\prime} \omega_{K}\left(\tau^{\prime}\right)}\right] \\
= & \frac{1}{2 \pi} \int_{-\infty}^{\infty} d \tau e^{i \Omega \tau} \sum_{K}\left(\frac{2 \pi \hbar c^{2}}{\omega_{K} V}\right)^{1 / 2}\left[\hat{a}_{K} e^{i\left(\epsilon_{K} \omega_{K} c / a\right) e^{-\epsilon_{K} a \tau / c}}\right. \\
& \left.+\hat{a}_{K}^{\dagger} e^{-i\left(\epsilon_{K} \omega_{K} c / a\right) e^{-\epsilon_{K} \omega_{K} \tau / c}}\right],
\end{aligned}
$$

where $\epsilon_{K}=|K| / K$. Since $\hat{a}_{K} \mid$ vacuum $\rangle=0$, only the $\hat{a}_{K}^{\dagger}$ terms in this expression contribute to the vacuum expectation value $\left\langle\hat{g}^{\dagger}(\Omega) \hat{g}\left(\Omega^{\prime}\right)\right\rangle$. Performing the integrals over $\tau$ as before, and using $\left\langle\hat{a}_{K} a_{K^{\prime}}^{\dagger}\right\rangle=\delta_{K K^{\prime}}$, we obtain

$$
\left\langle\hat{g}^{\dagger}(\Omega) \hat{g}\left(\Omega^{\prime}\right)\right\rangle=\left(\frac{c}{2 \pi a}\right)^{2}\left(\frac{2 \pi \hbar c^{2}}{V}\right)\left|\Gamma\left(\frac{i \Omega c}{a}\right)\right|^{2} e^{-\pi c \Omega / a} \sum_{K} \frac{1}{\omega_{K}}\left(\frac{\omega_{K} c}{a}\right)^{i \epsilon_{K}\left(\Omega-\Omega^{\prime}\right) c / a}
$$

where we use the fact that the sum over $k$ vanishes unless $\Omega-\Omega^{\prime}$. In fact we show in the Appendix that the sum over $K$ is $\left(2 V a / c^{2}\right) \delta\left(\Omega-\Omega^{\prime}\right)$, so that

$$
\left\langle\hat{g}^{\dagger}(\Omega) \hat{g}\left(\Omega^{\prime}\right)\right\rangle=\frac{\hbar c^{2}}{\pi a}\left|\Gamma\left(\frac{i \Omega c}{a}\right)\right|^{2} e^{-\pi c \Omega / a} \delta\left(\Omega-\Omega^{\prime}\right)=\frac{2 \hbar c / \Omega}{e^{2 \pi \Omega c / a}-1} \delta\left(\Omega-\Omega^{\prime}\right),
$$

which is identical to the thermal result Eq.(13) if we define the temperature by equation Eq.(1).

Note that the expectation value $\left\langle\hat{a}_{K} a_{K^{\prime}}^{\dagger}\right\rangle=\delta_{K K^{\prime}}$ involves the creation and annihilation operators of the accelerated observer and is taken with respect to the accelerated observer's vacuum, which is different from the vacuum seen by the nonaccelerated observer. This point is discussed more fully in Section VI.

\section{FERMI-DIRAC STATISTICS FOR DIRAC PARTICLES}

In the above we have considered a scalar field and have derived the Planck factor $\left(e^{\hbar \Omega / k T}-\right.$ $1)^{-1}$ indicative of Bose-Einstein (BE) statistics. We began with the standard plane-wave 
solutions of the form $\exp \left[i\left(K z \pm \omega_{K} t\right)\right]$ for the nonaccelerated Minkowski observer, and considered the time-dependent Doppler shifts as seen by the accelerated observer. For the spin-1/2 Dirac particles one would expect an analogous derivation to reproduce the Planck factor $\left(e^{\hbar \Omega / k T}+1\right)^{-1}$ indicative of Fermi-Dirac (FD) statistics.

We now show that this is indeed the case. Mathematically, the essential point involves the replacement $i \Omega c / a \rightarrow i \Omega c / a+1 / 2$ in the integrals in Eq.(7)- Eq.(9) ${ }^{14}$, and the relationship $|\Gamma(i \Omega c / a+1 / 2)|^{2}=\pi / \cosh (\pi \Omega c / a)^{9}$. Physically, this replacment arises from the additional spinor nature of the Dirac wave function over that of the scalar plane wave. In the case of a scalar field, only the phase had to be instantaneously Lorentz-transformed to the comoving frame of the accelerated observer. For non-zero spin, the spinor structure of the particles must also be transformed ${ }^{15}$, or "Fermi-Walker transported" 16 along a particle's trajectory to ensure that it does not "rotate" as it travels along the accelerated trajectory. This leads to a time-dependent Lorentz transformation of the Dirac bispinor of the form ${ }^{17} \hat{S}(\tau)=$ $\exp \left(\gamma^{0} \gamma^{3} a \tau / 2 c\right)=\cosh (a \tau / 2 c)+\gamma^{0} \gamma^{3} \sinh (a \tau / 2 c)$, where the $4 \times 4$ constant Dirac matrices are given by

$$
\gamma^{0}=\left(\begin{array}{cc}
1 & 0 \\
0 & -1
\end{array}\right), \quad \gamma^{3}=\left(\begin{array}{cc}
0 & \sigma_{z} \\
-\sigma_{z} & 0
\end{array}\right),
$$

and $\sigma_{z}=\operatorname{diagonal}(1,-1)$ is the usual $2 \times 2$ Pauli spin matrix in the $\mathbf{z}$ direction. Acting on a spin up state $|\uparrow\rangle=[1,0,1,0]^{T 18}, \hat{S}(\tau)$ gives $\hat{S}(\tau)|\uparrow\rangle=\exp (a \tau / 2 c)|\uparrow\rangle$. Thus, for the spin up Dirac particle we should replace the plane wave scalar "wave function" $\exp [i \varphi(\tau)]$ used in Eq.(7) with $\exp (a \tau / 2 c) \exp [i \varphi(\tau)]^{19}$. This leads to the replacement $i \Omega c / a \rightarrow i \Omega c / a+1 / 2$ in Eq.(8), and therefore the result

$$
\left|\int_{-\infty}^{\infty} d \tau e^{i \Omega \tau} e^{a \tau / 2 c} e^{i\left(\omega_{K} c / a\right) e^{a \tau / c}}\right|^{2}=\frac{2 \pi c}{\omega_{K} a} \frac{1}{e^{2 \pi \Omega c / a}+1} .
$$

Comparing Eq.(17) with Eq.(9) we note the change of sign in the denominator from -1 for BE statistics to +1 for FD statistics. We also note that the prefactor in Eq.(9) involves the dimensionless frequency $\Omega c / a$ while in Eq.(17) the prefactor involves the factor $\omega_{K} c / a$ (the argument of the exponential in the distribution function is still $\hbar \Omega / k T$ with the same Unruh temperature $T=\hbar a / 2 \pi k c)$. This is no cause for concern, since in fact a single Minkowski frequency $\omega_{K}$ is actually spread over a continuous range of accelerated (Rindler) frequencies 
$\Omega$ with peak centered at $\Omega=\omega_{K}{ }^{20}$. This allows us to replace $\omega_{K}$ by $\Omega$ in the final result. (This is explicitly evidenced by the delta function $\delta\left(\omega_{K}-\Omega\right)$ in Eq.(11) - Eq.(16) in the comparison of the thermal and accelerated correlation functions.)

For the case of the spin up Dirac particle, the more formal field-theoretic derivation of Section IV proceeds in exactly the same fashion, with the modification of the accelerated wave function from $\exp [i \varphi(\tau)] \rightarrow \exp (a \tau / 2 c) \exp [i \varphi(\tau)]$ and the use of anti-commutators $\left\{\hat{a}_{K}, a_{K^{\prime}}^{\dagger}\right\}=\delta_{K K^{\prime}}$ for the quantum-mechanical creation and annihilation operators instead of the commutators appropriate for scalar BE particles. For the correlation function we find

$$
\left\langle\hat{g}^{\dagger}(\Omega) \hat{g}\left(\Omega^{\prime}\right)\right\rangle=\frac{2 \hbar c / \omega_{K}}{e^{2 \pi \Omega c / a}+1} \delta\left(\Omega-\Omega^{\prime}\right)
$$

the FD analogue of Eq.(16).

\section{SUMMARY AND DISCUSSION}

In the usual derivation of the Unruh temperature Eq.(1), ${ }^{2,3,4,5,6}$ one solves the wave (or Dirac) equation for the field mode functions in the Rindler coordinates Eq.(4) and then quantizes them. Since the hyperbolic orbit of the accelerated observer is confined to the (right Rindler) wedge $z>|t|$ (with "mirror" orbits in the left Rindler wedge $z<-|t|$ ) it turns out that the vacuum seen by the accelerated observer in say, the right Rindler wedge is different than the usual Minkowski vacuum (defined for all $z$ and $t$ ) seen by the unaccelerated observer. The inequivalence of these vacua (and hence the Minkowski vs. Rindler quantization procedures ${ }^{21}$ ) is due to the fact that the right and left Rindler wedges are causally disconnected from each other. This can be easily seen by drawing a Minkowski diagram in $(z, t)$ coordinates and observing that light rays at $\pm 45^{\circ}$ emanating from one wedge do not penetrate the other wedge. Hence the Minkowski vacuum that the accelerated observer moves through appears to him as an excited state containing particles, and not as the vacuum appropriate for the right Rindler wedge. The Bose-Einstein distribution with Unruh temperature $T$ for scalar fields (Fermi-Dirac for Dirac fields) then arises by considering the expectation value of the number operator $\hat{a}_{R}^{\dagger} \hat{a}_{R}$ for the accelerated observer (in the right Rindler wedge) in the unaccelerated Minkowski vacuum $\left|0_{M}\right\rangle$, i.e. $\left\langle 0_{M}\left|\hat{a}_{R}^{\dagger} \hat{a}_{R}\right| 0_{M}\right\rangle \sim$ 
$(\exp (\hbar \Omega / k T) \pm 1)^{-1}$ (with upper sign for scalar fields and lower sign for Dirac fields). This is referred to as the Thermalization Theorem by Takagi ${ }^{5}$.

In this work we take a slightly different viewpoint ${ }^{22}$. For a scalar field, we first consider an unaccelerated Minkowski observer in a thermal state and find that the expectation value of a field correlation function is proportional to the Bose-Einstein distribution. We then consider the calculation of this correlation function again, but this time for an accelerated Rindler observer in his Rindler vacuum ${ }^{23}$ state $\left|0_{R}\right\rangle$, such that for a single mode, $\left\langle 0_{R}\left|\hat{a}_{R} \hat{a}_{R}^{\dagger}\right| 0_{R}\right\rangle=1$. The new feature now is that from his local stationary perspective, the accelerated observer perceives all Minkowski frequencies (arising from the the usual plane waves associated with Minkowski states) as time-dependent Doppler shifted frequencies.

The derivation presented in this work shows why quantum field fluctuations in the vacuum state are crucial for the thermal effect of acceleration: $\left\langle\hat{g}^{\dagger}(\Omega) \hat{g}\left(\Omega^{\prime}\right)\right\rangle$ is nonvanishing because the vacuum expectation $\left\langle\hat{a}_{K} \hat{a}_{K}^{\dagger}\right\rangle \neq 0$. But there's more to it than that, because $\left\langle\hat{a}_{K} \hat{a}_{K}^{\dagger}\right\rangle$ is also nonvanishing for an observer with $a=0$. For such an observer, however,

$$
\int_{-\infty}^{\infty} d \tau e^{i \Omega \tau} e^{i \int^{\tau} d \tau^{\prime} \omega_{K}\left(\tau^{\prime}\right)}=\int_{-\infty}^{\infty} d \tau e^{i\left(\Omega+\omega_{K}\right) \tau}=0
$$

for the case of scalar particles. In other words, the thermal effect of acceleration in our model arises because of the nontrivial nature of the quantum vacuum and the time-dependent Doppler shifts seen by the accelerated observer. For the case of Dirac particles, the essential new feature is the additional spinor structure of the wave function over that of the scalar plane wave. In order to keep the spin "non-rotating" in the comoving frame of the accelerated observer, the Dirac bispinor must be Fermi-Walker transported along the accelerated trajectory, resulting in an additional time-dependent Lorentz transformation. Formally, this induces a shifting of $i \Omega c / a \rightarrow i \Omega c / a+1 / 2$ in the calculation of relevant gamma function-like integrals, leading to the FD Planck factor.

In the following we briefly discuss the relationship of our correlation function to those used in the standard literature on this subject and point out a not widely appreciated curious subtlety relating details of the spatial Rindler mode functions (which we have ignored in our model) to the statistics of the noise spectrum seen by the accelerated observer.

In our model, we have not given any motivation for using the correlation function 
$\left\langle\hat{g}^{\dagger}(\Omega) \hat{g}\left(\Omega^{\prime}\right)\right\rangle$ aside from the fact that we could calculate it for a nonaccelerated observer in a thermal field and for a uniformly accelerated observer in vacuum and compare the results. It is easy to show that a harmonic oscillator with frequency $\omega_{0}$ and dissipation coefficient $\gamma$, linearly coupled to the field Eq.(10), reaches a steady-state energy expectation value

$$
\langle E\rangle \propto \int_{0}^{\infty} d \Omega \int_{0}^{\infty} d \Omega^{\prime} \frac{\left\langle\hat{g}^{\dagger}(\Omega) \hat{g}\left(\Omega^{\prime}\right)\right\rangle}{\left(\Omega-\omega_{0}-i \gamma\right)\left(\Omega^{\prime}-\omega_{0}+i \gamma\right)},
$$

which offers some motivation for considering $\left\langle\hat{g}^{\dagger}(\Omega) \hat{g}\left(\Omega^{\prime}\right)\right\rangle$. In fact, it can be shown that $\langle E\rangle=\left[e^{\hbar \omega_{0} / k T}-1\right]^{-1}$, which shows again that our accelerated observer acquires the characteristics appropriate to his being in a thermal field at the temperature $T=\hbar a / 2 \pi k c$.

In an extensive review of the Unruh effect, Takagi ${ }^{5}$ (Chapter 4) utilizes the quantum two-point correlation (Wightman) function $g_{W}\left(\tau, \tau^{\prime}\right) \equiv\left\langle 0_{M}\left|\hat{\phi}(\tau) \hat{\phi}^{\dagger}\left(\tau^{\prime}\right)\right| 0_{M}\right\rangle$ to determine the power spectrum of the vacuum noise seen by the accelerated observer for a scalar field via

$$
S(\Omega) \equiv \lim _{s \downarrow 0} \int_{\infty}^{\infty} e^{-i \Omega \tau-s|\tau|} g_{W}(\tau)
$$

which is very much in the spirit of our calculation. Here the field operator $\hat{\phi}(\tau)$ is expanded in terms of the Rindler mode functions and involve creation and annihilation operators for both the right and left Rindler wedges. Takagi shows the remarkable, though not widely known result, that for a scalar field in a Rindler spacetime of dimension $n$, $S_{n}(\Omega) \sim f_{n}(\Omega) /\left[\exp (\hbar \Omega / k T)-(-1)^{n}\right]$. For even-dimensional spacetimes (e.g. $n=2$ considered in this work, or the usual $n=4) S_{n}(\Omega)$ is proportional to the Bose-Einstein distribution function $[\exp (\hbar \Omega / k T)-1]^{-1}$, and essentially reproduces our equation Eq.(16) (up to powers of $\Omega c / a$, contained in the function $f_{n}(\Omega)$ ). However, for $n$ odd, $S_{n}(\Omega)$ is actually proportional to the Fermi-Dirac distribution $[\exp (\hbar \Omega / k T)+1]^{-1}$. For the case of Dirac particles the opposite is true, namely for even spacetime dimensions $S_{n}(\Omega)$ is proportional to the FD distribution and for odd spacetime dimensions $S_{n}(\Omega)$ is proportional to the BE distribution. This curious fact arises from the dependence of $S_{n}(\Omega)$ on two factors in its calculation. The first is the above mentioned Thermalization Theorem, namely that the number spectrum of accelerated (Rindler) particles in the usual nonaccelerated Minkowski vacuum is proportional to the BE distribution function for scalar fields and is proportional to the FD distribution 
function for Dirac fields. The second factor that switches the form of $S_{n}(\Omega)$ from BE to FD depends on the detailed form of the Rindler mode functions (see Takagi ${ }^{5}$, Chapter 4 for more complete details). Though the trajectory of the accelerated observer takes place in " $1+1$ " dimensions (i.e. the $(z, t)$ plane), the quantum field exists in the full $n$-dimensional spacetime, and thus $S_{n}(\Omega)$ ultimately depends of the form of the mode functions in the full spacetime. In spacetimes of even dimensions the number spectrum of Rindler particles in the Minkowski vacuum and the noise spectrum of the vacuum fluctuations (i.e., the response of the accelerated "particle detector") both depend on the same distribution function, and these two effects are often incorrectly equated.

In our simplified derivation we have bypassed this technicality by performing our calculations in " $1+1$ " dimensions (i.e., $n=2$ ). We have concentrated on the power spectrum of vacuum fluctuations as seen by a particle detector carried by the accelerated observer. We have shown that in " $1+1$ " dimensions the spectrum of fluctuations is proportional to the Bose-Einstein distribution function for scalar fields and to the Fermi-Dirac distribution for spin-1/2 fields, with the Hawking-Unruh temperature defined by Eq.(1). The dependence of the noise spectrum on these distribution functions is ultimately traced back to the timedependent Doppler shifts as seen by the accelerated observer as he moves through the usual nonaccelerated Minkowski vacuum. It is hoped that the simple calculations exhibited here are straightforward enough to give an intuitive understanding of the essential physical origin of the Hawking-Unruh temperature experienced by a uniformly accelerated observer.

Exercise for the student Discuss when the Hawking-Unruh temperature from Eq.(1) would become physically detectable by utilizing the expression $a=G M / r^{2}$ for the gravitational acceleration of a test mass at a distance $r$ from a mass $M$, and determining $T$ at the surface of the earth, the sun and a Schwarzschild black hole.

\section{Appendix}

Converting the sum over $K$ to an integral, we have, for $K>0$,

$$
\sum_{K>0} \frac{1}{\omega_{K}}\left(\frac{\omega_{K} c}{a}\right)^{i \epsilon_{K}\left(\Omega-\Omega^{\prime}\right) c / a}=\frac{V}{2 \pi} \int_{0}^{\infty} d K \frac{1}{\omega_{K}}\left(\frac{\omega_{K} c}{a}\right)^{i\left(\Omega-\Omega^{\prime}\right) c / a} .
$$


Letting $x=\log \left(\omega_{K} c / a\right)$, we can write this as

$$
\frac{V}{2 \pi c} \int_{-\infty}^{\infty} d x e^{-i x\left(\Omega-\Omega^{\prime}\right) c / a}=\frac{V a}{c^{2}} \delta\left(\Omega-\Omega^{\prime}\right)
$$

The same result is obtained for the sum over $K<0$, so that the sum over all $K$ is $\left(2 V a / c^{2}\right) \delta\left(\Omega-\Omega^{\prime}\right)$.

\section{Acknowledgements}

Thanks go to H. Fearn, D. McMahon, G. J. Milburn E. Mottola, M. O. Scully and M. Wolinsky for brief discussions relating to acceleration in vacuum.

1 S. W. Hawking, Nature 248, 30 (1974).

2 W. G. Unruh, Phys. Rev. D14, 870 (1976).

3 P. C. W. Davies, J. Phys. A8, 609 (1975).

4 There are many papers on the subject. See, for instance, P. Candelas and D. W. Sciama, Phys. Rev. Lett. 38, 1372 (1977); T. H. Boyer, Phys. Rev. D21, 2137 (1980) and D29, 1089 (1984);

D. W. Sciama, P. Candelas, and D. Deutsch, Adv. Phys. 30, 327 (1981).

5 An extensive review is given by S. Takagi, Prog. Theor. Phys. 88, 1 (1986).

6 N. D. Birrell and P. C. W. Davies, Quantum Fields in Curved Space (Cambridge University Press, N. Y., 1982).

7 W. Rindler, Am. J. Phys 34, 1174 (1966).

8 I. S. Gradshteyn and I. M. Ryzhik, Table of Integrals, Series, and Products (Academic Press, N. Y., 1980). We have used integrals Sec. 3.761.4 and Sec. 3.761.9 $\int_{0}^{\infty} x^{\mu-1} \sin (a x) d x=\frac{\Gamma(\mu)}{a^{\mu}} \sin \left(\frac{\mu \pi}{2}\right)$ and $\int_{0}^{\infty} x^{\mu-1} \cos (a x) d x=\frac{\Gamma(\mu)}{a^{\mu}} \cos \left(\frac{\mu \pi}{2}\right)$ respectively, in the combination of the second plus $i$ times the first. Taken together, both integrals have a domain of definition $a>0,0<\operatorname{Re}(\mu)<1$. In Eq.(8) we have $\mu=i \Omega c / a$ with $\operatorname{Re}(\mu)=0$. The integrals can be regularized and thus remain valid in the limit $R e(\mu) \rightarrow 0$ as can be seen by adding a small imaginary part $-i \epsilon a / c, \epsilon>0$ to the frequency $\Omega$ so that now $\mu \rightarrow \mu^{\prime} \equiv \mu+\epsilon$ and 
$0<\operatorname{Re}\left(\mu^{\prime}\right)=\epsilon<1$ is strictly in the domain of definition of the integrals. In the limit of $\epsilon \rightarrow 0$ we have $\frac{\Gamma(\mu+\epsilon)}{a^{\mu+\epsilon}} e^{i(\mu+\epsilon) \pi / 2} \rightarrow \frac{\Gamma(\mu)}{a^{\mu}} e^{i \mu \pi / 2}$ and thus obtain the same values for the integrals as if we had just set $\operatorname{Re}\left(\mu^{\prime}\right)=0$ to begin with. This occurs because the standard integral form of the gamma function $\Gamma(z)=\int_{0}^{\infty} d t e^{-z} t^{z-1}, \operatorname{Re}(z)>0$ can be analytically continued in the complex plane and in fact remains well defined, in particular, for $\operatorname{Re}(z) \rightarrow 0, \operatorname{Im}(z) \neq 0$, which is our case in Eq.(8). See for e.g. J.T. Cushing, Applied Analytical Mathematics for Physical Scientists, 343, John Wiley and Sons, NY (1975).

9 Reference [8], Sec. 8.332.

10 A related, though much more involved derivation of the Unruh effect based on a similar subsitution can be found in L. Pringle, Phys. Rev. D 39, 2178 (1989). One can justify this subsitution from the form $\varphi=\int k_{\mu}(x) d x^{\mu}$ of the phase of a quantum mechanical particle in curved spacetime. See L. Stodolsky Gen. Rel. Grav. 11, 391 (1979) and P.M. Alsing, J.C. Evans and K.K. Nandi, Gen. Rel. Grav. 33, 1459 (2001); gr-qc/0010065.

11 A similar derivation in terms of Doppler shifts appears in the appendix of H. Kolbenstvedt, The principle of equivalence and quantum detectors, Eur. J. Phys. 12, 119-121 (1991). T.

Padmanabhan and coauthors have have also derived Eq.(9) by similarly considering the power spectrum of Doppler shifted plane waves as seen by the accelerated observer, see K. Srinivasan, L. Sriramkumar and T. Padmanabhan, Plane waves viewed from an accelerated frame: Quantum physics in a classical setting, Phys. Rev. D 56, 6692-6694 (1997); T. Padmanabhan, Gravity and the Thermodynamics of Horizons, gr-qc/0311036.

12 When we later convert a sum over $K$ to an integral, we obtain the Lorentz-invariant measure $d K / \omega_{K}$ as a consequence of the $1 / \sqrt{\omega_{K}}$ in Eq.(10). This eliminates the need to explicitly transform this quantity in Eq.(14), for instance.

13 Since we use a one-dimensional model the factor $V / 2 \pi$ appears instead of the more familiar $V /(2 \pi)^{3}$. In other works, our volume $V$ here is really just a length.

14 Using the same gamma function integrals as in Reference [8] above, we will now have for the Dirac case $\mu=i \Omega c / a+1 / 2$, with $\operatorname{Re}(\mu)=1 / 2$ clearly in their domain of definition $0<\operatorname{Re}(\mu)<1$. 
15 S. Weinberg, Gravitation and Cosmology (Wiley, N.Y.,1972), pp. 365-70.

16 C. W. Misner, K.S. Thorne and J.A. Wheeler, Gravitation (W.H. Freeman and Co., San Francisco, 1973), Chapter 6, pp. 163-176,

17 Reference [5], p. 101, and P. Candelas and D. Deutsch, Proc. R. Soc. Lond. A 362, 251 (1978). This result can be understood as follows. If the observer was travelling at constant velocity $v$ in the $\mathbf{z}$ direction, we would Lorentz transform the spinor in the usual special relativstic way via the operator $\hat{S}(v)=\exp \left(\gamma^{0} \gamma^{3} \xi / 2\right)$ where $\tanh \xi=v / c$; see J.D. Bjorkin and S.D Drell Relativistic Quantum Mechanics, (McGraw-Hill, Inc, N.Y., 1964), p28-30. ¿From Eq.(3) we have $v / c=\tanh (a \tau / c)$ so that $\xi=a \tau / c$, yielding the spinor Lorentz transformation to the instantaneous rest frame of the accelerated observer.

18 For simplicity, we have chosen our spin up wave function as an eigenstate of $\hat{S}(\tau)$ with eigenvalue $\exp (a \tau / 2 c)$. The exact spatial dependence of the accelerated (Rindler) spin up wave function is more complicated than this simple form, though both have the same zero bispinor components. See W. Greiner, B. Müller and J. Rafelski, Quantum Electrodynamics in Strong Fields (Springer, N.Y., 1985), Chapter 21.3, pp. 563-567; M. Soffel, B. Müller and W. Greiner Phys. Rev. D22, 1935 (1980).

19 The spinor Lorentz transformation $\hat{S}(\tau)$ does not mix spin components. Thus, for e.g., a spin up Minkowski state remains a spin up accelerated (Rindler) state. We can therefore drop the constant spinor $|\uparrow\rangle$ from our calculations and retain the essential, new time-dependent modification $\exp (a \tau / 2 c)$ to the plane wave for our Dirac "wave function."

20 Reference [5], Section 2, in particular Eqs. (2.7.4) and (2.8.8).

21 S.A. Fulling, Phys. Rev. D 7, 2850 (1973); Aspects of Quantum Field Theory in Curved Space-Time, Cambridge Univ. Press, N.Y. (1989).

22 This viewpoint is also taken in a different derivation of the Unruh-Davies effect in P.W. Milonni, The Quantum Vacuum, (Academic Press, NY, 1994), p60-64.

23 The unaccelerated Minkowski vacuum $\left|0_{M}\right\rangle$ is unitarily related to the Rindler vacuum $\left|0_{R}\right\rangle \otimes\left|0_{L}\right\rangle$ via $\left|0_{M}\right\rangle=\hat{S}(r)\left|0_{R}\right\rangle \otimes\left|0_{L}\right\rangle$ where $\hat{S}(r)$ is the squeezing operator $\hat{S}(r)=\exp \left[r\left(\hat{a}_{R} \hat{a}_{L}-\hat{a}_{R}^{\dagger} \hat{a}_{L}^{\dagger}\right)\right]$. Here the subscripts $R$ and $L$ denote the right $(z>|t|)$ and left 
$(z<-|t|)$ Rindler wedges, respectively, and $\left|0_{R}\right\rangle$ is the Fock state of zero particles in the right Rindler wedge and $\left|0_{L}\right\rangle$ is the Fock state of zero particles in the left Rindler wedge. Since these right and left Rindler wedges of Minkowski spacetime are causally disconnected from eachother, the creation and annihilation operators $\hat{a}_{R}^{\dagger}, \hat{a}_{R}$ and $\hat{a}_{L}^{\dagger}, \hat{a}_{L}$ live in the right and left wedges respectively and mutually commute with with each other, i.e. $\left[\hat{a}_{R}, \hat{a}_{L}^{\dagger}\right]=0$, etc. ..

Since, physical states that live in the right wedge have zero support in the left wedge (and visa versa), they are described by functions solely of the operators $\hat{a}_{R}^{\dagger}, \hat{a}_{R}$ appropriate for the right wedge, i.e. $\left|\Psi_{R}\right\rangle=f\left(\hat{a}_{R}, \hat{a}_{R}^{\dagger}\right)\left|0_{R}\right\rangle \otimes\left|0_{L}\right\rangle=\left|\psi_{R}\right\rangle \otimes\left|0_{L}\right\rangle$. It is in this sense that we can speak of $\left|0_{R}\right\rangle$ as the "vacuum" for the right Rindler wedge, and similarly $\left|0_{L}\right\rangle$ as the "vacuum" for the left Rindler wedge. 